\title{
LA EVOLUCIÓN DE LA SEXUALIDAD PREPUBERAL Y ADOLESCENTE: DIFERENCIAS EN AFECTOS Y CONDUCTAS ENTRE VARONES Y MUJERES
}

Félix López, Noelia Fernández-Rouco, Rodrigo J. Carcedo, Asia Kilani y María Isabel Blázquez Universidad de Salamanca flopez@usal.es - fernandezrouco@usal.es - rcarcedo@usal.es

Recibido: 07-08-2009

Aceptado: 14-04-2010

\section{Resumen}

Este trabajo aborda y profundiza en el debate sobre la existencia de un posible doble patrón en afectos y conductas sexuales entre varones y mujeres prepúberes y adolescentes. En este sentido, diferentes estudios plantean la existencia de este doble patrón sexual, si bien es verdad que se pone de manifiesto de forma más evidente con respecto a los afectos que con respecto a las conductas. Estudios más recientes incluso plantean que varones y mujeres mantienen el mismo tipo y frecuencia de conductas sexuales, especialmente a medida que tienen más edad. Además, existen diferentes planteamientos sobre el posible origen de este doble patrón sexual. Finalmente, entendemos que las diferencias, cuando existen, se relacionan tanto con factores biológicos como socioculturales.

Palabras clave: sexualidad, adolescentes, sexo/género, afectos y conductas. 


\begin{abstract}
This work deals with and go deeply in the debate about the presence of a double standard in sexual feelings and behaviors between prepuberal and adolescent boys and girls. Different studies have found a double sexual standard with respect to sexual feelings and behaviors. Recent studies point out that male and female adolescents have the same type and frequency of sexual behaviors, especially when they are older. In addition, diverse approaches offer different explanations for this double standard. Finally, we point out that male and female differences, when they appear, are related to both biological and sociocultural factors.
\end{abstract}

Key words: sexuality, adolescents, sex/gender, sexual feelings, and sexual behavior.

El objetivo principal de este trabajo es profundizar en el debate sobre la existencia de un posible doble patrón en afectos y conductas sexuales entre varones y mujeres prepúberes y adolescentes, como así muestran diferentes estudios. No obstante, este patrón parece más evidente con respecto a los afectos que con respecto a las conductas. En cuanto a las conductas y su frecuencia, algunos autores señalan un mayor grado de similitud, especialmente a medida que los niños y las niñas avanzan en edad.

\title{
1. Sexo, afecto y diversidad
}

La aparición, a lo largo de la filogénesis humana, de los sexos, ha dado lugar a hombres y mujeres. Distintos en cuanto machos o hembras y distintos en cuanto personas: individualidad, identidad sexual y rol de género. Todos los seres sexuados pertenecen a una especie y, dentro de ella, tienen una identidad sexual, la de machos o la de hembras. Estos dos hechos hacen que los sexos sean semejantes, con la especie y con los miembros que comparten identidad sexual; pero también que sean muy 
diferentes en razón del sexo, del rol de género y de su individualidad. Parece elemental, pero lo olvidamos continuamente.

La reproducción sexual trajo otras grandes novedades, muy importantes para el tema que nos ocupa: funciones distintas en la reproducción (el macho y la hembra tienen morfología, fisiología y conducta sexual, emociones y afectos sexuales (deseo, atracción y enamoramiento). Se hizo necesario el deseo y la atracción sexual (sin ellos, el macho y la hembra no se buscarían) y el placer sexual (para asegurar la repetición de la conducta de apareamiento por el alto poder reforzante del placer). Desear aparearse, sentir atracción por la otra identidad sexual y gozar con el apareamiento era una condición necesaria para la supervivencia de las especies sexuadas. En las especies que la cría naciera indefensa e incapaz de alimentarse, la naturaleza tuvo que asegurar la supervivencia con otros afectos sociales: los cuidados (de uno o los dos progenitores) y la impronta o apego (de la cría). El enamoramiento parece un lujo añadido en la especie humana que facilita el que estén juntos, al menos durante un tiempo.

Resulta así que el dimorfismo en machos y hembras desencadena o conlleva, una serie de cambios que afectan al cuerpo, a su funcionamiento, especialmente en la reproducción, a las emociones y afectos sexuales y, por último, a los afectos sociales.

A pesar de que estos hechos dieron origen a una gran diversidad, de especies y, dentro de ellas, de machos y hembras, así como también a una diversidad individual, durante mucho tiempo los seres vivos se relacionaron sexualmente y se aparearon de forma preprogramada e instintiva, como decíamos antes. Estos programas eran, por un lado específicos (los miembros de cada especie eran muy parecidos en su conducta) y, por otro, propios de los machos o de las hembras (los machos se parecían mucho entre sí y también las hembras). Dada determinada edad, sexo, condición hormonal y situación, era fácil predecir la conducta del macho y de la hembra.

Pero, por un lado, la sexualidad está menos programada en la especie humana, hasta el punto de que en ella los individuos pueden tomar decisiones, decir sí o no a una oportunidad de conducta sexual, son libres sexualmente de hacer esto o aquello; por otro lado, en las especies sociales y culturales como la humana, la conducta sexual ha sido 
regulada socialmente y su conducta y afectos han estado y están sujetos a leyes y construcciones sociales diferentes. En efecto, según la cultura, la conducta sexual es regulada de forma diferente, y conductas y afectos son interpretados de diferente manera. Por tanto, además de posibles diferencias biológicas y psicológicas, de base, es evidente que las culturas influyen en la forma que los sexos y los individuos viven la sexualidad y los afectos.

En efecto, a lo largo de la filogénesis humana, en algún momento, la conducta sexual deja de estar preprogramada de forma estereotipada e instintiva, para hacerse sujeto de decisión e interpretación, es decir, humana. Este camino hacia la libertad y el simbolismo es la verdadera razón que nos hace diferentes a cada uno de nosotros, en cuanto personas únicas dentro de la especie humana y en cuanto manera única también de ser hombre o mujer. ¿Y lo común?: es evidente que lo común es mucho e importante, el ser persona y el ser hombre o mujer. Hay además otras muchas variables: el momento histórico, el país, la generación, los padres que tenemos, los iguales que nos rodean, los medios de comunicación y las experiencias personales, entre otros muchos factores, contribuyen a nuestra diversidad de comportamientos sexuales, por un lado, y a nuestra semejanza, en cuanto compartamos estas variables, por otro.

\section{Diversidad de base: Anatomía, fisiología y roles sexuales distintos en la reproducción}

Entre las diferencias anatómicas y fisiológicas más importantes hay que destacar los siguientes hechos:

- El pene del varón es más externo que el clítoris de la mujer. Esta diferencia anatómica puede tener gran importancia facilitando el descubrimiento de la excitación sexual y el aprendizaje de la masturbación (Baldwin y Baldwin, 1997). Fisiológicamente, además, la erección del pene es una condición para el coito, hecho que ha facilitado que el pene se convierta en 
símbolo de poder y causa de ansiedad para el varón, especialmente si se concede al coito el único protagonismo en una relación sexual. El ocultamiento del clítoris no impide, en todo caso, que un número importante de mujeres descubran su significado sexual; significado sexual que ha llevado a algunas culturas, contrarias al placer sexual de la mujer, a incluir el rito de su eliminación.

- El hombre mantiene desde la pubertad capacidad reproductora y en cada eyaculación suele disponer de miles de espermatozoides que vuelven a reproducirse, prácticamente, sin límite; su capacidad reproductora, por otra parte, no está sujeta a ciclos de temporalidad después de la pubertad, ni a cambios equivalentes a la menopausia. Su compromiso con la reproducción se limita, desde el punto de vista biológico, a la aportación del espermatozoide. En este hecho biológico encuentra la teoría evolucionista la razón de la mayor disponibilidad del varón a aceptar y buscar el sexo ocasional, sin implicaciones emocionales y sin compromisos formales. En definitiva, desde el punto de vista biológico, el varón puede tener muchas conductas sexuales, disfrutar del placer y no está vinculado a ninguna consecuencia. La responsabilidad con la crianza es un hecho social para el varón (Buss, 1999).

- La mujer tiene un número amplio, pero limitado de óvulos, está sujeta a ciclos que regulan su reproducción; a la vez que esta capacidad tiene un final cronológico definido, la menopausia. La mujer tiene un compromiso biológico mucho mayor con la reproducción: aportar el óvulo, gestar durante nueve meses, parir y amamantar. También es probable que el "sistema de cuidados" (tendencia a ocuparse incondicionalmente de la cría) esté biológicamente más preprogramado en la mujer. Estos hechos biológicos explicarían, según la misma teoría evolucionista, que la mujer sea más selectiva buscando varones dispuestos a comprometerse con la crianza y con recursos para defender, sostener, etc., a la familia. Esta sería la base biológica que explica la tendencia 
de la mujer a dar más peso a las implicaciones emocionales, al compromiso y a las cualidades del varón como protector y padre.

Otras diferencias anatómicas y fisiológicas afectan también a la sexualidad (la sexuación cerebral, las diferencias hormonales, las diferencias en la respuesta sexual humana, etc.), pero es más difícil concretar cómo finalmente regulan la manera de ser del hombre o mujer y están mucho más sujetas a diferencias individuales entre personas. De todas ellas, una de las más evidentes es que el varón tiene una capacidad limitada de eyaculaciones, en determinados periodos de tiempo, y un periodo refractario después de cada orgasmo, más o menos acentuado, en relación con la edad y otros factores. En el coito, depende también en el varón, claro está, de la capacidad de erección.

La mujer, por el contrario, no tiene limitaciones marcadas en relación a las conductas coitales, ni periodo refractario propiamente dicho y, por supuesto, no depende de la capacidad eréctil. Hay grandes discusiones sobre una posible mayor simplicidad o capacidad para dar una respuesta sexual más fácil o más mecánica (expresiones bien inadecuadas) en el caso del varón, mientras la mujer tendría una activación sexual más compleja y más dependiente de factores emocionales, sociales y culturales, teniendo, por tanto, una sexualidad más maleable y compleja (Andersen, Cyranowski y Aarestad, 2000; Baumeister, 2000; Baumeister, Catanese, Campbell y Tice, 2000). Pero todas estas diferencias están muy sujetas a influencias de factores, históricos, culturales y sociales, por un lado, y también a grandes diferencias individuales entre hombres y entre mujeres, por otro. 


\section{Segunda diversidad: doble patrón en la conducta y en los afectos sexuales}

\section{1. ¿Puede hablarse de un doble patrón sexual y afectivo entre prepúberes?}

En una investigación anterior hemos podido encontrar un doble patrón sexual en los niños y niñas prepúberes (López, Del Campo y Guijo, 2003), como otros autores (Friedrich, Standfort, Oosteveen y Cohen-Kettenis, 2000; Sandnabba y Ahberg, 1999; Sandnabba, Santtila, Wannas y Kook, 2003).

¿Qué aspectos conductuales comparten los prepúberes?: Sin duda, los más importantes. Ambos, niños y niñas, tienen la fisiología del placer sexual activable; es decir, pueden excitarse y aprender a excitarse con determinadas conductas.

Ambos sexos están muy interesados por conocer e interpretar numerosos aspectos de la sexualidad. La práctica totalidad de niños y niñas hacen preguntas explícitas, especialmente antes de los seis años, tienen conductas de observación y se muestran intrigados por saber qué significan ciertas cosas que hacen los adultos.

Un número relativamente importante de prepúberes se excita sexualmente y lleva a cabo conductas por motivación de placer; incluso un número de prepúberes, aunque en este caso más reducido, parece haber tenido experiencias que los adolescentes recuerdan como orgásmicas (Bullough, 2004; Davis, Glaser y Kossof, 2000; Kaeser, Disalvo y Moglia, 2000; Larsson y Svedir, 2002; López et al., 2003; Standfort y Cohen-Kettenis, 2000; Vizcarral, Balladares, Candía, Lepe y Sildivia, 2004; Volpert, 2000).

¿A pesar de estas semejanzas, puede hablarse de un doble patrón en las conductas sexuales prepuberales? Creemos que sí, tomados los niños y las niñas como grupos diferentes; pero ninguna de las conductas sexuales deja de ser hecha por algunos niños o por algunas niñas, por lo que la posible especificidad de las conductas más frecuentes en un sexo, no es ajena a un número de personas del otro sexo. Visto de manera radical e individual, no hay nada específico de los niños o de las niñas. ¿Cuáles serían estas diferencias entre el grupo de niñas y el grupo de niños? (López et al., 2003): 
- Los niños en general tienen más conductas sexuales que las niñas. Hecho que confirman los adolescentes (por recuerdos), los padres y los educadores (por observaciones). En general, los niños tienen más frecuencia de conductas. Pero las niñas superan a los niños en las siguientes: Masturbarse con un objeto (conducta muy condicionada por la anatomía del niño -poco apropiada para ello- y de la niña), imitar besos, acariciar, conducta de seducción, enamoramientos.

Se trata de conductas con un significado sexual, pero también con contenidos afectivos más explícitos. En todas las otras conductas los niños superan a las niñas, aunque, en proporciones distintas. Las diferencias en la frecuencia de masturbación están entre las más claras.

- Las niñas tienen conductas sexuales más motivadas por su interés en los juegos sexuales, mientras los varones están comparativamente más motivados directamente por el placer.

- Los niños se excitaron y llegaron comparativamente más al orgasmo, que las niñas, con base en los recuerdos adolescentes.

Las niñas se sintieron con mayor frecuencia culpables que los niños.

Por tanto, si atendemos a los datos, parece que la sexualidad de las niñas es más afectivo-relacional que la de los niños; mientras que la de éstos está más directamente relacionada con la excitación y el placer sexual, aspectos ambos, que como hemos dicho, no están ausentes en el otro sexo necesariamente. Estos resultados son congruentes con otras investigaciones anteriores y posteriores (Friedrich, Grambsch, Broughton, Kuiper y Beilke, 1991; Friedrich et al., 2000; Sandnabba y Ahberg, 1999; Sandnabba et al., 2003). Por ejemplo, en una de las más recientes, se demuestra que los 
niños prepúberes tienen más conductas sexuales explícitas, piensan más y tienen más prisa en tener relaciones sexuales que las niñas (Ballester y Gil, 2006).

\subsection{Semejanzas y diferencias en la adolescencia}

Es indudable que en la pubertad chicos y chicas se alejan aun más en su anatomía y en su fisiología sexual. Las funciones en la reproducción, nueva capacidad alcanzada, son distintas, como veíamos. La anatomía se marca y especifica (tanto en los órganos sexuales primarios, como en los secundarios). La cronología de la pubertad es diferente, como también es sabido. Entre todas estas diferencias destaca el mayor incremento de los niveles de testosterona en los varones (que llega a ser entre 7 y 13 veces más alto que en las mujeres), lo que puede revestir gran importancia dada la indudable relación entre testosterona, activación y deseo sexual, aunque no se trate de una relación mecánica y simple.

Estas diferencias anatómicas, fisiológicas y de cronología de la pubertad pueden tener grandes implicaciones para la conducta sexual. ¿Pero puede hablarse de un doble patrón sexual en las actitudes y conductas sexuales de adolescentes y jóvenes?

Hasta hace unos años, este doble patrón era muy evidente: los pensamientos, los sentimientos y las conductas estaban claramente diferenciados entre los chicos y las chicas. Los chicos, comparativamente con las chicas, eran más liberales y erotofílicos, desde el punto de vista de las actitudes, estaban más dispuestos a valorar positivamente las conductas sexuales y a involucrase en ellas, incluso sin poner la condición de la mediación de compromisos y afectos. De hecho, los chicos accedían antes, como grupo, a las diferentes experiencias sexuales, tenían más parejas sexuales y valoraban más la actividad sexual. Las chicas interiorizaban más la moral conservadora, eran más erotofóbicas y, de hecho, tenían menos conductas sexuales, menos número de parejas y valoraban menos la actividad sexual, etc. Este doble patrón solía resumirse diciendo que los hombres buscaban más actividad sexual y las mujeres más afecto (Giordano, 
Longmore y Manning, 2006). ¿Qué queda de estos patrones entre los adolescentes y jóvenes actuales?

En una primera aproximación, centrada solo en el estudio de las conductas, se pone de manifiesto que las cosas han cambiado mucho, acercando o diluyendo estos padrones:

- La mayor parte de los chicos y chicas tienen opiniones liberales y sentimientos ertofílicos. De hecho, por ejemplo, les parece legítimo masturbarse, si así lo desean; admiten las relaciones prematrimoniales; no ponen como condición necesaria el compromiso de pareja, ni los afectos, aunque estos dos últimos aspectos juegan, de hecho, un rol importante. Lo que consideran que debe regular las relaciones es la libertad de cada uno para decir sí o decir no, siendo el mutuo consenso, para la mayoría, la única condición verdaderamente necesaria. Es decir, se consideran libres para decidir, concediendo poco peso a la moral, el estado civil, etc.

Esto es hoy cierto para los chicos y también para las chicas, que opinan de forma muy similar y tienen conductas, cada vez más semejantes (Navarro, 2002; Usillos, 2002).

- $\quad$ Si tomamos como referencia el inicio de las relaciones coitales (entre los 15 y 18 años, según las muestras usadas en las distintas investigaciones) y otras manifestaciones de la conducta sexual, las diferencias entre chicos y chicas son mucho menores; en algunas muestras incluso no se aprecian (Op. cit.).

Pero, desde un segundo punto de vista, más complejo y profundo, las cosas no han cambiado tanto y se mantienen elementos de los patrones tradicionales, por un lado, y de un doble patrón más estable, vinculado a los dos sexos, como hemos visto en los prepuberes (Zurbriggen y Morgan, 2006). ¿Qué elementos actuales nos permiten hablar de un doble patrón? 
- Desde el punto de vista de las conductas (Baldwin y Baldwin, 1997; Buss, 1999; Oliver y Hyde, 1993):

- La conducta de masturbación la práctica un número mucho mayor de chicos (entre el ochenta y noventa por ciento frente al cincuenta o sesenta por ciento de chicas), haciéndolo, además, con mayor frecuencia.

- Los varones están más dispuestos al sexo ocasional, es decir, a tener relaciones sexuales sin especiales afectos o compromisos. Incluso, aunque ambos sexos lleguen a tener actitudes y conductas cada vez más cercanas, casi todos los estudios confirman la mayor disponibilidad del varón para el sexo ocasional.

- En relación al tipo de conductas sexuales, las mujeres encuentran más gusto por la comunicación, las caricias y los juegos afectivo-sexuales, mientras los varones, suelen buscar más directamente la conducta coital. Por eso en las primeras manifestaciones sexuales (besos, caricias, etc.), las diferencias chicos y chicas son menores (Baldwin y Baldwin, 1997; Navarro, 2002; Oliver y Hyde, 1993) que en cuanto a las relaciones coitales.

- Otra forma de reconocer los patrones es desde el punto de vista de las quejas que unos y otras suelen hacer. En este sentido, las chicas suelen quejarse del machismo, la falta de delicadeza, y el hecho de que vayan demasiado directos y rápidos al coito. También de un menor interés afectivo y comunicación; y de que si se muestran demasiado abiertas y activas sexualmente las interpretan mal y las tienen por "desvergonzadas". Los chicos se quejan más de la resistencia de las chicas para aceptar tener relaciones sexuales, de que le dan demasiadas vueltas a las cosas, etc. Naturalmente estas quejas no las asumen todos, ni se refieren a todos los chicos y chicas. 
- Las mujeres asocian la actividad sexual más a los afectos, la intimidad y el compromiso que los varones (Giordano et al., 2006). De hecho suelen tener menos parejas y dar un significado afectivo y social más fuerte a la pareja que han tenido o tienen. Por ejemplo, las primeras parejas suelen tener más contenido emocional y social para las mujeres que para los hombres. Son también más las mujeres que ponen la condición, más o menos explícita, de los afectos, de que sus relaciones sexuales tengan lugar en un contexto amoroso y, de hecho, su actividad sexual está más vinculada al hecho de tener pareja. "Las mujeres ponen de manifiesto más sentimientos negativos en relación con la conducta sexual que tiene lugar entre individuos con poca implicación emocional, aunque ambos, hombres y mujeres, se muestran más parecidos cuando están en una relación de pareja” (Hill, 2002: 213).

- En las conductas sexuales más ocasionales, también es más frecuente que las mujeres acaben atribuyéndole más contenido afectivo y tienen más dificultades para que la experiencia sexual haya sido solo eso: un encuentro para compartir el placer sexual (Baldwin y Baldwin, 1997; Browing, Hatfield, Kessler y Levine, 2000; Feiring, 1996; Hendrick y Hendrick, 1992; Spira y Bajos, 1993; Taris y Semin, 1997).

- Los varones tiene fantasías con contenidos sexuales más explícitos que las mujeres, especialmente cuando éstas no han tenido o están empezando a tener relaciones sexuales. Es frecuente que las mujeres, cuando fantasean un encuentro con un hombre, lo contextualicen más en un sistema de relaciones más amplio, sean más escénicas y románticas, en comparación con los varones. Aunque, como en todos los aspectos, un número relativamente importante de mujeres tienen fantasías sexuales muy explícitas, especialmente después de tener experiencias sexuales placenteras (García, Fernández y Rico, 2005). Por otra parte, cuando están en pareja, los varones suelen fantasear más con otras 
mujeres, aunque las mujeres que han tenido numerosas experiencias sexuales lo hacen con una frecuencia similar a los hombres (Hicks y Leitenberg, 2001).

- Los varones ven, se excitan y disfrutan más con los contenidos eroticopornográficos que las mujeres. Las mujeres están más interesadas por contenidos románticos o erótico-sexuales, más que pornográficos. Parece que la sexualidad del varón es más visual y táctil, mientras que la de la mujer, siendo también visual y táctil atiende a estímulos y factores relacionales más complejos (Zurbriggen y Morgan, 2006). No obstante, algunos autores cuestionan estas diferencias y, sobre todo, su origen (Baumeister, 2000).

- Las mujeres tienen mayor percepción de riesgos, en el caso de prácticas sexuales de riesgos, están más interesadas y dispuestas a prevenirlos, postergar el coito si no se dispone de condón, por ejemplo, asistir a cursos de promoción de la salud y el bienestar, participar en discusiones y actividades de grupo, etc. Finalmente, parece que están más dispuestas a mejorar su salud y a contribuir a la salud de los demás. Los varones tienden a asumir más riesgos en las relaciones sexuales y están menos dispuestos a invertir esfuerzos en prevenirlos (Navarro, Barbera y Reig, 2003; Usillos, 2002).

- Las mujeres suelen aceptar mejor a determinadas minorías como las personas homosexuales, transexuales, etc. Estas diferencias no aparecen tanto cuando se trata de dar opiniones (Navarro et al., 2003; Oliver y Hyde, 1993), como cuando están involucrados en relaciones familiares o de amistad con personas que pertenecen a estas minorías. Esto es especialmente evidente cuando ellas son madres, hermanas o amigas de quienes pertenecen a estas minorías, frente a padres, hermanos y amigos, los cuales suelen aceptarlas peor.

- Las mujeres con más frecuencia que los hombres, tienen su iniciación sexual con una pareja mayor que ellas.

- Donde el patrón se hace particularmente claro es en las relaciones agresorvíctima (Zurbriggen y Morgan, 2006). Los agresores son casi siempre varones, 
tanto el abuso sexual infantil, como en el acoso y la violación, mientras las víctimas son con más frecuencia mujeres. Aunque, en relación con las víctimas de abusos sexuales, también lo son un grupo importante de niños (en torno al $10 \%$ ); este número es claramente inferior al de las niñas víctima de abusos (en torno al $20 \%$ de chicas). Esto demuestra que es mucho más probable que los varones empleen la violencia, la coerción o el engaño, para imponer prácticas sexuales a las mujeres o a los hombres, buscando en unos casos la satisfacción sexual y, en otros, fines más perversos, como la demostración de poder, etc.

Como la sexualidad pertenece al reino de la libertad y el ser humano tiene capacidad de dar significados, ocurre que con frecuencia la actividad sexual se usa con fines no fisiológicamente previstos, por decirlo así. Los varones es más probable que la usen para demostrarse a sí mismos que son capaces (su miedo a la impotencia es especialmente característico), que pueden conquistar y obtener así aprobación de otros varones e, incluso, lo que es especialmente grave, que pueden doblegar, dominar, hacer sufrir, etc., a la mujer, chica o niña o niño con la que tienen relaciones. Por eso, usan con más frecuencia la presión, la coerción y la violencia que las mujeres (Zurbriggen y Morgan, 2006; García et al., 2005).

- En último lugar, las mujeres utilizan con más frecuencia la sexualidad para demostrase y demostrar que son o siguen siendo atractivas, conseguir ser aceptadas o queridas por su pareja (López, Del Campo y Guijo, 2003).

Estos patrones descritos se mantienen en la vida adulta básicamente de manera similar (López et al., 2003), por lo que hay una cierta estabilidad en ellos desde la primera infancia hasta la vejez, más allá de los cambios propiamente evolutivos. Finalmente, como señalaban estudios ya clásicos (Gilligan, 1982, Maccoby, 1990) y confirman estudios actuales (García et al., 2005; Giordano et al., 2006; Zurbriggen y Morgan, 2006), las mujeres son más convencionales en cuanto a las conductas y valores 
sexuales, están más motivadas por las relaciones interpersonales, los afectos y por conductas de caricias, abrazos, besos, etc.; mientras los hombres están más motivados por la actividad sexual convencional y no convencional, especialmente por la masturbación y el sexo ocasional, tienen un estilo de interacción menos íntimo y están menos dispuestos a adoptar compromisos. Pero las cosas están cambiando, especialmente entre los más jóvenes, ¿podemos reconocer hoy en los niños y principalmente en los adolescentes estos patrones?

A esta pregunta debería dar contestación futuras investigaciones realizadas en nuestro país, como la que nos encontramos desarrollando en estos momentos. Datos preliminares nos llevan a pensar que este doble patrón aún existe, especialmente en lo que a los afectos sexuales se refiere, que sin embargo tiende a desaparecer o igualarse entre ambos sexos en cuanto a las conductas sexuales. En otras palabras, chicos y chicas adolescentes no mostrarían tantas diferencias en cuanto a las conductas sexuales que practican (e.g., besos, caricias, sexo oral, coito vaginal, etc.), si bien estás diferencias estarían presentes en cuanto a sus afectos sexuales, mostrando los chicos un mayor nivel de deseo y atracción sexual.

\section{Conclusión: ¿A qué se debe este doble patrón que se encuentra a lo largo del ciclo vital, manteniéndose en la actualidad en menores prepúberes y en adolescentes?}

Desde nuestro punto de vista, a la luz de las investigaciones y los resultados expuestos, concluimos que las alternativas explicativas son tres:

En primer lugar, las diferencias podrían tener una base biológica. A favor de esta interpretación está el hecho transcultural de las diferencias y el que se mantengan a lo largo del ciclo vital. Esta base podría explicarse, al menos en parte, por las diferencias anatómicas de los genitales y las diferencias hormonales, ya comentadas. Baldwin y Baldwin (2000), por ejemplo, consideran que las diferencias prepuberales se deben a factores biológicos, como son el tamaño y ubicación del pene (facilita el 
descubrimiento del placer y la masturbación) y el clítoris.

Los evolucionistas (Buss, 1999) también son partidarios de esta interpretación, pero ponen el énfasis en la diferente función biológica que hombres y mujeres tienen en la reproducción, lo que a lo largo de la historia de la especie habría llevado a los hombres a aceptar mejor que las mujeres el sexo ocasional y la búsqueda despreocupada del placer, mientras las mujeres aprendieron a ser más selectivas y a valorar más otras cosas para no tener que cargar solas con los hijos.

Una segunda interpretación pone el énfasis en la explicación cultural. Según ésta, los hombres dominantes (como ocurre en otras especies cercanas) instrumentalizaron a las mujeres sometiéndolas a sus intereses sexuales o a un rol de "madres de los hijos". Por eso reprimieron su sexualidad, la eliminación del clítoris sería la versión más dramática, e impusieron una doble moral en la que los varones se sentían libres para tener conductas sexuales con diferentes mujeres, mientras sometían a sus mujeres a una monogamia forzada. El hecho de que las niñas, chicas y mujeres se sientan más culpables teniendo menos fantasías, menos intereses, menos deseos y menos conductas sexuales que los niños, chicos y hombres, sería la demostración más fehaciente (López, et al., 2003). Todo ello ha provocado que las mujeres se repriman más, tengan menos conductas sexuales, busquen menos el placer con pensamientos intereses, deseos y conductas, mientras, por el contrario, se sienten más culpables.

Una tercera hipótesis sostiene que hay razones biológicas, evolucionistas y culturales, aunque sea difícil indicar el peso de cada una de ellas y la interacción entre ellas. Ésta es la que nosotros consideramos más razonable, pero con una salvedad muy importante: las razones evolucionistas han dejado de tener un verdadero peso original (otra cosa es su peso ancestral, aún presente) porque las mujeres tienen menos dependencia económica y social del hombre y pueden tener actividad sexual sin embarazo por lo que no tiene sentido que no valoren sus intereses y deseos sexuales más originales, con independencia de la reproducción. En definitiva, el placer para ellas, ha dejado de tener riesgos. 
Finalmente, en paralelo a estos cambios científicos que permiten el control de la fecundidad y los cambios sociales que hacen que muchas mujeres sean autónomas económicamente del varón, así como la pérdida de peso de las ideas religiosas, se ha producido el hecho más relevante sobre la regulación de la sexualidad, culturalmente hablando: las mujeres se consideran (porque tienen) con el derecho a decidir cuándo tener relaciones sexuales. Ésta es la verdadera revolución que no ha hecho más que empezar, por lo que concluimos diciendo que las bases evolucionistas y culturales del doble patrón están claramente en trance de desaparecer, ¿tendrán en este nuevo contexto las bases biológicas (anatómicas, cerebrales y hormonales) el poder de mantener algunos aspectos de estos patrones en el futuro? Esta es la cuestión. 


\section{BIBLIOGRAFÍA}

- Andersen, B. L.; Cyranowski, J. M. y Aarestad, S. (2000): "Beyond artificial, sexlinked distinctions to conceptualize female sexuality". En Psychological Bulletin, $\mathrm{n}^{\circ}$. 126, pp. 380-384.

- Baldwin, J. D. y Baldwin, J. L. (1997): “Gender differences in sexual interest”. En Archives of Sexual Behavior, no. 26, pp. 181-210.

- Ballester, R. y Gil, M. (2006): "La sexualidad en niños de 9 a 14 años". En Psicothema, vol. 18, nº 1, pp. 25-30.

- Baumeister, R. F. (2000). "Gender differences in erotic plasticity: The female sex drive as socially flexible and responsive". En Psychological Bulletin, nº. 126, pp. 347374.

- Baumeister, R. F.; Stillwell, A. M. y Heatherton, T. F. (1994): “Guilt: An interpersonal approach”. En Psychological Bulletin, vol. 115, n. 2, pp. 243-267.

- Baumeister, R. F.; Catanese, K. R.; Campbell, W. K., y Tice, D. M. (2000): "Nature, culture, and explanations for erotic plasticity". En Psychological Bulletin, no. 126, pp. 385-389.

- Browning, J. R., Hatfield, E., Kessler, D. y Levine, T. (2000): “Sexual motives, gender and sexual behaviour". En Archives of Sexual Behavior, n. 29, pp. 135-143.

- Bullough, V. (2004): "Children and adolescents as sexual beings: a historical overview". En Child and Adolescent Psychiatric Clinics of North America, nº 13, pp. 447-459.

- Buss, D. M. (1999): The evolution of desire: Strategies of human mating. Nueva York: Basic Book.

- Davies, S.; Glaser, D. y Kossof, R. (2000): “Children's sexual play and behaviour in pre-school settings: staff's perceptions, reports and responses". En Child Abuse and Neglect, $\mathrm{n}^{\text {o. }}$ 24, pp. 1.329-43. 
- Feiring, C. (1996): “Concepts of romance in 15-year-old adolescent”. En Journal of Research on Adolecence, $n^{o}$. 6, pp. 181-200.

- Friedrich, W. N.; Grambsch, P.; Broughton, D.; Kuiper, J. y Beilke, R. (1991): "Normative sexual behavior in children". En Pediatrics, n'. 88, pp. 456-464.

- Friedrich, W. N.; Sandfort, T.; Oostveen, J. y Cohen-Kettenis, P. (2000): "Cultural differences in sexual behavior: 2-6 year old Dutch and American children”. En Journal of Psychology and Human Sexuality, nº. 12, pp. 117-29.

- García, E.; Fernández, P. y Rico, R. A. (2005): “Género y sexo como variables moduladoras del comportamiento sexual en jóvenes universitarios". En Psicothema, vol. $17, \mathrm{n}^{\circ} .1$, pp. 49-56.

- Gilligan, C. (1982): In a different voice: Psychological theory and women's development. Cambridge, MA: Harvard University Press.

- Giordano, P. C.; Longmore, M. A. y Manning, W. D. (2006): “Gender and the meanings of adolescent romantic relationships: A focus on boys". En American Sociological Review, vol. 71, nº. 2, pp. 260-287.

- Hendrick, S. y Hendrick, C. (1992): Romantic Love. Londres: Routledge.

- Hill, G. (2002): “Gender, relationship stage and sexual behaviour”. En The Journal of Sex Research, $\mathrm{n}^{\circ} .39$, pp. 228.240.

- Kaeser, F.; Disalvo, C. y Moglia, R. (2000): "Sexual behaviors of young children that occur in schools". En Journal of Sex Education and Therapy, n. 25, pp. 277-85.

- Larsson, I. y Svedin, C. (2002): “Teachers' and parents' report on 3 to 6 years old children's sexual behavior: a comparison". En Child Abuse and Neglect, n. 26, pp. 24766.

- López, F.; Del Campo, A. y Guijo, V. (2003): "Prepuberal sexuality". En European Journal of Medical Sexology, $\mathrm{n}^{\circ} .12$, pp. 49-65.

- Maccoby, E. E. (1990): “Gender and relationship: A developmental account”. En American Psychologist, $\mathrm{n}^{\circ}$. 45, pp. 513-520.

- Navarro, E. (2002): Adolescencia y sexualidad. Diferencias de género en la iniciación sexual. Tesis doctoral: Universidad de Valencia. 
- Oliver, M. J. y Hyde, J. S. (1993): "Gender differences in sexuality: A meta-analysis". En Psychological Bulletin, no . 114, pp. 29-51.

- Sandfort, T. y Cohen-Kettenis, P. (2000): "Sexual behavior in Dutch and Belgian children as observed by their mothers". En Journal of Psychology and Human Sexuality, $\mathrm{n}^{\mathrm{o}}$. 12, pp. 105-115.

- Sandnabba, N. y Ahlberg, C. (1999): "Parents' attitudes and expectations about children's cross-gender behavior". En Sex Roles, nº 40, pp. 249-63.

- Sandnabba, N.; Santtila, P.; Wannäs, M. y Krook, K. (2003): ”Age and gender specific sexual behaviours in children”. En Child Abuse and Neglect, n. 27, pp. 579-605.

- Spira, A. y Bajos, N. (1993): Les comportements sexuales en France. Paris: La Documentation Francaise.

- Taris, T. W. y Semin, G. R. (1997): "Gender as a moderator of the effects of the love motive and relational context on sexual experience". En Archives of Sexual Behavior, n'. 26, pp. 159-180.

- Usillos, S. (2002): Actitudes, creencias y conductas sexuales de riesgo. Tesis doctoral: Universidad del País Vasco.

- Vizcarral, M. B. et al. (2004): “Conducta sexual durante la infancia en estudiantes chilenos”. En Psicothema, no. 16, pp. 58-63.

- Volbert, R. (2000): "Sexual knowledge of preschool children". En Journal of Psychology and Human Sexuality, nº. 12, pp. 5-26.

- Zurbriggen, E. y Morgan, E. (2006): "Who wants to marry a millionaire? Reality dating television programs, attitudes toward sex and sexual behaviour". En Sex Roles, vol. $54, n^{\circ} .1-2$, pp. 1-17. 